\title{
Superconducting resonator used as a phase and energy detector for linac setup
}

\author{
Nikolai R. Lobanov \\ The Department of Nuclear Physics, Research School of Physics and Engineering, \\ Australian National University, Canberra ACT 2601, Australia
}

(Received 9 May 2016; published 19 July 2016)

\begin{abstract}
Booster linacs for tandem accelerators and positive ion superconducting injectors have matured into standard features of many accelerator laboratories. Both types of linac are formed as an array of independently-phased resonators operating at room temperature or in a superconducting state. Each accelerating resonator needs to be individually set in phase and amplitude for optimum acceleration efficiency. The modularity of the linac allows the velocity profile along the structure to be tailored to accommodate a wide range charge to mass ratio. The linac setup procedure, described in this paper, utilizes a superconducting resonator operating in a beam bunch phase detection mode. The main objective was to derive the full set of phase distributions for quick and efficient tuning of the entire accelerator. The phase detector was operated in overcoupling mode in order to minimize de-tuning effects of microphonic background. A mathematical expression was derived to set a limit on resonator maximum accelerating field during the crossover search to enable extracting unambiguous beam phase data. A set of equations was obtained to calculate the values of beam phase advance and energy gain produced by accelerating resonators. An extensive range of linac setting up configurations was conducted to validate experimental procedures and analytical models. The main application of a superconducting phase detector is for fast tuning for beams of ultralow intensities, in particular in the straight section of linac facilities.
\end{abstract}

DOI: 10.1103/PhysRevAccelBeams.19.072801

\section{INTRODUCTION}

Superconducting, or room temperature, short independently-phased accelerating structures have been in use for many decades in heavy ion booster linacs [1], positive ion injectors and high energy ion implanters [2]. The linac covers the velocity range $\beta=\mathrm{v} / \mathrm{c}$ spanning from 0.01 to 0.3 . Each accelerating resonator needs to be individually tuned in phase and amplitude for maximum acceleration and transmission efficiency. The short accelerating structures permit the velocity profile along the structure to be optimized to accommodate a wide range of charge to mass ratio. The buncher and rebuncher resonator electric field amplitude and phase also have to be set correctly.

A typical linac booster is the superconducting linac operating at the Australian National University (ANU) Heavy Ion Accelerator Facility (HIAF). The HIAF comprises a $15 \mathrm{MV}$ electrostatic accelerator followed by a superconducting linac booster as shown in Fig. 1. A bunched beam is obtained using a single or three frequency grid buncher operating at $1 / 16^{\text {th }}$ sub-harmonic of the linac frequency of $150 \mathrm{MHz}$. The buncher is positioned at the low energy (LE) of the 14UD accelerator. The beam

Published by the American Physical Society under the terms of the Creative Commons Attribution 3.0 License. Further distribution of this work must maintain attribution to the author(s) and the published article's title, journal citation, and DOI. bunches have a typical width of 1.5 ns FWHM with a bunching efficiency $\sim 25 \%$ at single frequency. The efficiency is about $50 \%$ when operating in three frequency mode. The beam is further compressed to $\sim 50-100 \mathrm{ps}$ wide by the superconducting buncher " $\mathrm{C} 1$ " made up of a $\beta=0.1$ Quarter Wave Resonator (QWR).

The twelve $\beta=0.1$ lead tin electroplated split loop resonators (SLR) currently installed in module cryostats "C2" mean that only double terminal stripped ions from the 14UD with mass below $\sim 70$ amu match adequately the resonator gap separation. Heavier ions have a velocity at injection into the linac of $\beta<0.06$, limiting its performance due to transit time factor (TTF) mismatching. The timeenergy lens, TEL, a beta 0.1 QWR, "C3", serves as the energy homogenizer for return of the beam to the target area TA1. Alternatively it works as rebuncher for optimal time resolution of beam delivered to the linac target lines TA2.

Two main factors limit 14UD performance due to double terminal stripping operation: (a) limited beam intensity and range of beams available from the ion sources and (b) restriction on transmission and high loading associated with heavy beams. For example, the beam loading limits the maximum intensity of injected beam to $2.0 \mu \mathrm{A}$ at LE end. Normally with double terminal stripping and single frequency bunching one can produce a few $\mathrm{nA}$ beam injected into the linac. A specialized sensitive diagnostics equipment is required to tune the linac with such low intensity beam. 
The setting of the bunching and acceleration phase of the accelerating resonators calls for measurement of crossover phases and identification of the type of crossover, bunching or debunching. It is accomplished by employing a few different techniques. First, it can be completed by energy measurement with semiconductor or gas detector, as for a given field amplitude of the resonator, the phase determines the energy gain or loss of accelerated beam. Second, it is realized by interpreting the beam profile monitor (BPM) trace [3]. The BPM should be installed after a bending magnet. In the case of HIAF, the BPM is located in the middle of a 180 degree achromat A2, shown in Fig. 1. The energy dispersion at this location allows observation of the beam energy affected by each successive resonator. A special large bore BPM is used for this procedure. The third technique employs a fast Faraday cup. It provides a direct view of the time structure of the beam [4] using an appropriate broadband amplifier and a sampling oscilloscope. The time width of the bunch can also be measured with a silicon surface barrier detector. A more sophisticated application employs a superconducting resonator to detect the arrival time of a beam bunch $[5,6]$.

This paper builds on our prior study [6] to develop an analytical model for calculation of the bunch phase advance and energy gain during acceleration and to define major experimental constraints. It has been organized into three sections. The first section outlines the general concept of the linac tuning procedure at very low sub-nA beam intensities, based on a superconducting cavity as a phase detector. The goal is to set up linac resonators for effective acceleration and determination of energy gain after each successive acceleration. The second section presents key

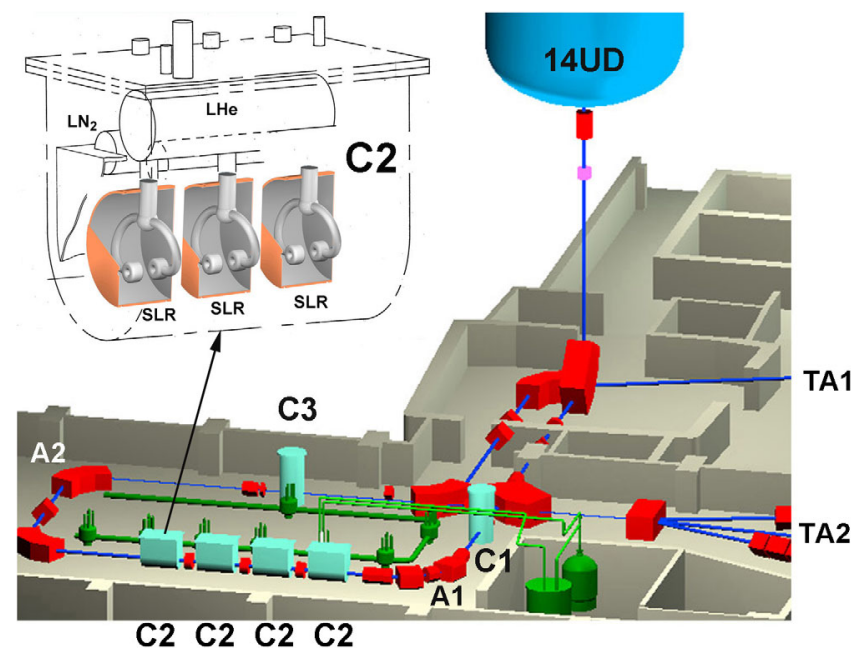

FIG. 1. Layout of the superconducting linac booster. The main components are: two target areas TA1 and TA2; the superbuncher $\mathrm{C} 1$; split loop resonator (SLR) modules $\mathrm{C} 2$ and the time-energy lens C3; 90 degrees achromatic bend A1 and 180 degrees achromat A2. The insert in the left top corner shows a rendered image of a cryostat module housing three SLR. experimental results based on the new technique. The data have been systematically collected during recent linac operations. Finally, the third section presents the interpretation of linac tune results and defines limitations of the new technique.

\section{METHODS}

\section{A. Superconducting cavity as a phase and time of flight detector}

A phase detector based on rf cavities has been developed for booster accelerating facilities and stand-alone linacs. The sensitivity $S$ is defined as the rf voltage peak to peak $V_{p p}$ per unit beam current $I_{b}$ produced at the external load $r$, or $S=2\left(R_{0} r\right)^{1 / 2} T(\beta)$, where $R_{0}$ is the shunt impedance and $T(\beta)$, is the transit time factor [7]. The sensitivity of room temperature resonators is in the order of $V_{p p} / I_{b} \sim$ $10 \mu \mathrm{V} / \mathrm{nA}$ of ion beam allowing operation at a minimum of a few nA of average beam current. The sensitivity $S$ can be increased by (a) maximizing the shunt impedance $R_{0}$ of the resonator and (b) implementing high impedance $r$ coupling of the resonator to the preamplifier [8].

In this specific example of beam phase detection mode for SLRs, the surface charge oscillates along the ring between the beam drift tubes. With microwave studio (MWS) electric $E$ and magnetic $H$ field distributions were calculated as shown in Fig. 2.

The peak surface electric field occurs at the end of each drift tube. The use of a superconducting cavity in phase detection mode results in very high sensitivity $S_{\mathrm{sc}}$ due to its very high shunt impedance $R_{0 \mathrm{sc}}$ compared to a room temperature resonator $R_{0 \mathrm{RT}}$. In the case of superconducting lead-tin film, the sensitivity is up by a factor of $S_{\mathrm{sc}} / S_{\mathrm{RT}}=$ $\left(R_{0 \mathrm{sc}} / R_{0 \mathrm{RT}}\right)^{1 / 2}=\left(R_{s \mathrm{Cu} 300 \mathrm{~K}} / R_{s \mathrm{PbSn} 4 \mathrm{~K}}\right)^{1 / 2} \sim\left(3.2 \times 10^{-3} /\right.$ $\left.100 \times 10^{-9}\right)^{1 / 2}=179$, where $R_{s \mathrm{Cu} 300 \mathrm{~K}}=3.2 \mathrm{~m} \Omega$ is the surface resistance of copper at $300 \mathrm{~K}$ and $R_{s \mathrm{PbSn} 4 \mathrm{~K}}=$ $100 \mathrm{n} \Omega$ is the low field surface resistance of $\mathrm{PbSn}$ film at $4.2 \mathrm{~K}$.

The unloaded quality factor $Q_{0}$ of our electroplated SLR is just above $3 \times 10^{8}$ resulting in a very low frequency bandwidth of $\Delta f_{\mathrm{BW}}=2 \pi f_{0} / Q_{0} \sim 3 \mathrm{~Hz}$ at an eigenfrequency of $f_{0}=150 \mathrm{MHz}$. A fundamental problem with

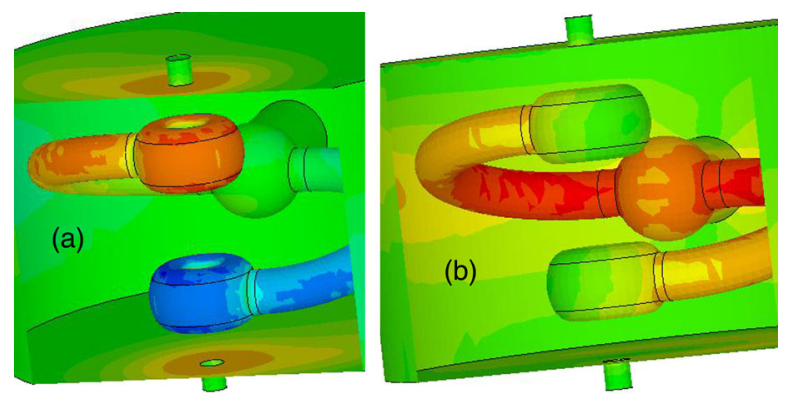

FIG. 2. Low frequency phase detecting mode for distributions of $E$-field (a) and $H$-field (b) in a SLR. 
superconducting resonators is the variation of resonant frequency caused by mechanical vibrations, including ground motion, vacuum pumps, refrigerator and boil off gas pressure variations. The RMS spectrum of microphonics is typically greater than the cavity bandwidth, preventing the direct use of beam induced signals for phase detection.

Finite element structures analyses (STRAND7), was used to identify the intrinsic mechanical resonance of the SLR. Four dominant frequencies were found, see Fig. 3, as previously identified by a stroboscopic technique [1].

The first two are a torsion and bending mode of the loop, where the drift tubes move in the same (Mode 1) and opposite (Mode 2) direction along the beam axis with frequencies $f_{1}=47 \mathrm{~Hz}$ and $f_{2}=48 \mathrm{~Hz}$. The other two are a bending mode of the loop, where the drift tubes move in the plane perpendicular to the beam axis in the same (Mode 3) and opposite (Mode 4) direction, and have frequencies $f_{3}=55 \mathrm{~Hz}$ and $f_{4}=56 \mathrm{~Hz}$. Since the loops move in the same direction for Modes 1 and 3, their frequencies are slightly lower compared to those for corresponding Modes 2 and 4 due to the contribution of the supporting column. Quite low resonance frequencies indicate poor stiffness of the SLR's loops.

Mechanical changes of the shape and eigenfrequency of the superconducting resonator, caused by pondermotive effects and by microphonic background, are the major contributors to amplitude and phase jitter. However for a cavity operating in phase detection mode at low rf power the pondermotive effects may be neglected. Typically the measured microphonic frequency error of a SLR in our module cryostat is below $3.5 \mathrm{~Hz}$ RMS. The application of the mechanical tuner by a single step may increase it up to $12 \mathrm{~Hz}$ RMS. Similarly, initiation of liquid nitrogen $\mathrm{LN}_{2}$ transfer introduces an additional shaking up to $8 \mathrm{~Hz}$ RMS. Therefore during phase measurements the operation of the mechanical tuner is avoided and $\mathrm{LN}_{2}$ refill is disabled.

The simplest technique to minimize detuning effects of microphonics is overcoupling of the rf pickup port, which in turn results in widening of the loaded bandwidth and lower sensitivity. For example, in order to widen the loaded
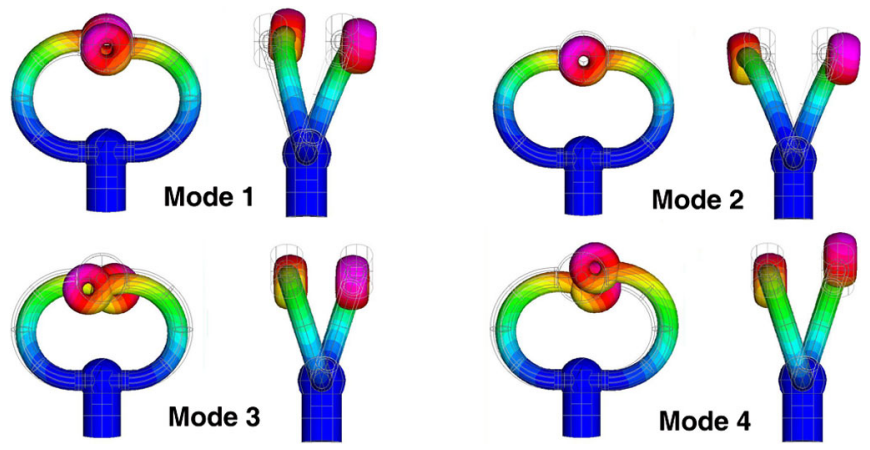

FIG. 3. The four lowest mechanical modes in SLR identified using Strand7. bandwidth $\Delta f_{L}$ of the SLR to an acceptable value of $\sim 100 \mathrm{~Hz}$ to allow overlapping microphonic bandwidth $\Delta f_{\mathrm{bw}}$, the coupling constant should be set to a value $\beta=\Delta f_{L} / \Delta f_{\text {bw }}+1=101$. The loaded quality factor $Q_{L}=Q_{0} /(1+\beta) \sim 10^{6}$ still remains much higher than $Q_{0}$ for the same SLR operating at room temperature.

The complete experimental setup, exploiting a superconducting SLR as a beam phase detector, is shown in Fig. 4.

The HP Model 8405A Vector Voltmeter measures amplitudes and phase simultaneously. The instrument has a sensitivity of $100 \mu \mathrm{V}$ full scale with phase resolution of $0.1^{\circ}$. Loading of the SLR is minimized by the high input impedance of the probes $(0.1 \mathrm{M} \Omega$ shunted by $2.5 \mathrm{pF})$. Phase meter electronic section of Vector Voltmeter HP8405A is as follows: resolution $0.1^{\circ}$ at any phase angle; phase accuracy $1.5^{\circ}$ at single frequency with equal voltages at Channels "A" and "B".

For operation as a phase detector the SLR is overcoupled to a loading constant $\beta \sim 101$ by driving its coupler to the predefined position with the stepper motor. In addition, the resonator frequency is to be retuned for operation at low rf power. The main cause of static frequency shift $\Delta f_{\text {stat }}$ of an accelerating resonator operating at high rf power is caused by electrostatic force according to equation $\Delta f_{\text {stat }}=K E_{\text {acc }}^{2}$, where $E_{\text {acc }}$ is accelerating gradient and radiation pressure constant $K \sim-100$ for a SLR. Indeed the measured range on-line static shifts was as high as -(60-100) $E_{\text {acc }}^{2} \mathrm{~Hz}$ over twelve SLRs. The dispersion of static shift may be due to variation of quality of balancing of the $E$-field profile. By substituting $E_{\text {acc }}=3.0 \mathrm{MV} / \mathrm{m}$ and $K=-100$ one can calculate $\Delta f_{\text {stat }}=-900 \mathrm{~Hz}$. The second cause of frequency shift is increased surface temperature of the superconductor at elevated $\mathrm{rf}$ power.

The channel "A" probe is connected to output of the linac master oscillator. Channel " $\mathrm{B}$ " probe is connected to the SLR inductive variable coupler. The phase recorder output provides a DC voltage proportional to the phase meter in the range \pm 180 degrees. Both amplitude and phase outputs are routed to the data logger.

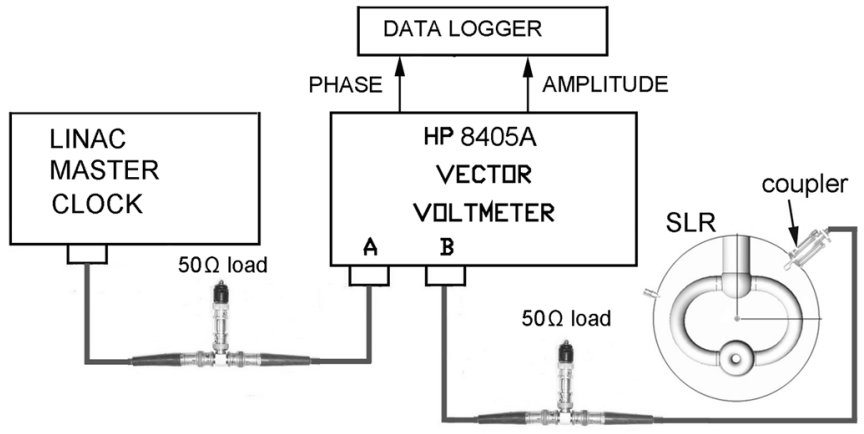

FIG. 4. Experimental setup utilizing a superconducting SLR as a beam phase detector. 


\section{B. Experimental constraints for unambiguous determination of resonator crossover phase}

The resonator crossovers phase occurs two times during the rf cycle when the rf field amplitude crosses zero value. The crossover phase information, obtained from the phase detector, is used to accurately determine the optimum acceleration phase of the resonator being tuned. In the experimental setup shown in Fig. 4, the vector voltmeter measures the magnitude of a reference and resonator voltage and the difference in phase between the voltages. The magnitude and phase of the reference signal are kept constant during crossover search. The level of beaminduced signal depends on beam defocusing due to varying beam energy. The phase of the beam-induced signal in respect to the reference phase is influenced by variation of the transit time of the beam bunch (due to energy gain/loss) and beam steering, caused by the accelerating resonator during its phase reference ramp.

Let us assume for simplicity that the phase of the accelerating resonator at crossover point with respect to the reference signal is set to zero degrees. If the transit time variation due to acceleration or deceleration exceeds the value $\Delta t_{\text {tr }}> \pm T / 2= \pm 1 / 2 f$, where $T$ is the rf period and linac central frequency $f=150 \mathrm{MHz}$, the phase measurement of the beam bunch $\Psi_{\text {acc }}=\Psi_{\text {dec }}= \pm 2 \pi f \Delta t_{\text {tr }}$ becomes uncertain outside the \pm 180 degrees measurement range. This makes crossover determination uncertain. There are two simple procedures to extract unambiguous phase data.

The first approach is based on extraction of bunch phase information by combining the information from phase detection measurements with BPM data as described in [9]. The approximate determination of the crossover phase is achieved by interpreting the BPM trace in the middle of the 180 degree achromat A2, shown on the left side of the Fig. 1. The energy dispersion at this location has allowed observation of the energy of the beam as it is affected by each successive resonator. The crossover phase and its type, bunching $-90^{\circ}$ or debunching $+90^{\circ}$, is inferred by variation of the accelerating resonator reference phase from $-180^{\circ}$ to $+180^{\circ}$ and briefly identifying phases with zero energy dispersion. After that, the measurement of the crossover phase is done at high resolution with the superconducting SLR operating in phase detection mode.

The second approach is to set a constraint on the resonator accelerating field and the corresponding energy gain in order to limit the bunch transit time between the cavity and phase detector to well below $\Delta t_{\text {tr }}< \pm T / 2$. The energy gain or loss introduced by the linac resonator is calculated by the equation

$$
\Delta E=q E_{\mathrm{acc}} L_{\mathrm{SLR}} \cos \left(\Delta \Psi_{\mathrm{acc}}\right) T_{(\beta)},
$$

where $q$ is the ion beam charge state; $E_{\text {acc }} \sim 3 \mathrm{MV} / \mathrm{m}$ is an average accelerating field produced at $6 \mathrm{~W}$ rf absorbed power; $L_{\mathrm{SLR}}=0.221 \mathrm{~m}$ is the active length of the SLR;
$\Delta \Psi_{\text {acc }}$ is the resonator field phase deviation from $0^{\circ}$ (the crest phase of rf standing wave in accelerating resonator) and transit time factor $T_{(\beta)}$. As a first approximation, the velocity of an ion with atomic mass $M$ and energy $E_{[\mathrm{MeV}]}$ is given by expression:

$$
v=c(2 E / 939 M)^{0.5}=0.046 c(E / M)^{0.5},
$$

where $c$ is the speed of light in vacuum and $E_{p, n} \sim$ $939 \mathrm{MeV} / \mathrm{c}^{2}$ is the rest mass of proton or neutron. The first-order differentiation of Eq. (2) is

$$
d v=\left(0.046 c / 2(E M)^{0.5}\right) d E,
$$

The transit time $t_{\text {tr }}$ of a beam bunch, traveling between the accelerating resonator and the phase detector SLR, separated by the distance $L$ is $t_{\mathrm{tr}}=L / v$. During tuning of a linac resonator the transit time varies since the bunch energy is changed by $\Delta E$, according to Eq. (1). The variation of transit time $\Delta t_{\text {tr }}$ is calculated from the differential equation $d t_{\mathrm{tr}}=-\left(L / v^{2}\right) d v$. The negative sign indicates that transit time decreases as velocity increases. By combining the last equation with Eqs. (2) and (3), one can arrive at the following expression which is valid if the energy variation $\Delta E \ll E$ :

$$
\Delta E=\left(-0.092 c E^{1.5} / L M^{0.5}\right) \Delta t_{\mathrm{tr}}
$$

Substituting (1) into (4) and assuming $\cos \left(\Delta \Psi_{\max }\right)=1$ at $\Delta \Psi_{\max }=0$ one now arrives at the maximum accelerating gradient $E_{\text {acc } \max }$ corresponding to $\Delta t_{\mathrm{tr}}= \pm T / 2= \pm 1 / 2 f$

$$
E_{\text {acc max }}=0.046 c E^{1.5} /\left(f q L_{\mathrm{SLR}} L T_{(\beta)} M^{0.5}\right)
$$

To obtain unambiguous beam phase data, the accelerating field of the resonator during crossover search is to be kept below the maximum value estimated by expression (5).

\section{Calculation of the bunch phase advance and energy gain}

The accuracy of expression (4) is better than $1.5 \%$ providing the relative energy gain is $\Delta E / E_{0}<0.05$. The variation of transit time $\Delta t_{\text {tr }}$ causes the alteration or advance in bunch phase measured by the phase detector according to formula

$$
\Delta \Psi_{\mathrm{det}}=2 \pi f \Delta t_{\mathrm{tr}}
$$

Substituting (4) into (6) gives the following equation for bunch phase advance

$$
\Delta \Psi_{\mathrm{det}[\mathrm{rad}]}=-k \Delta E / E,
$$

where $k$ is calculated using the following equation $k_{\text {[rad }]}=21.74 \pi(f L / c)(M / E)^{0.5}$. The calculated plot of 


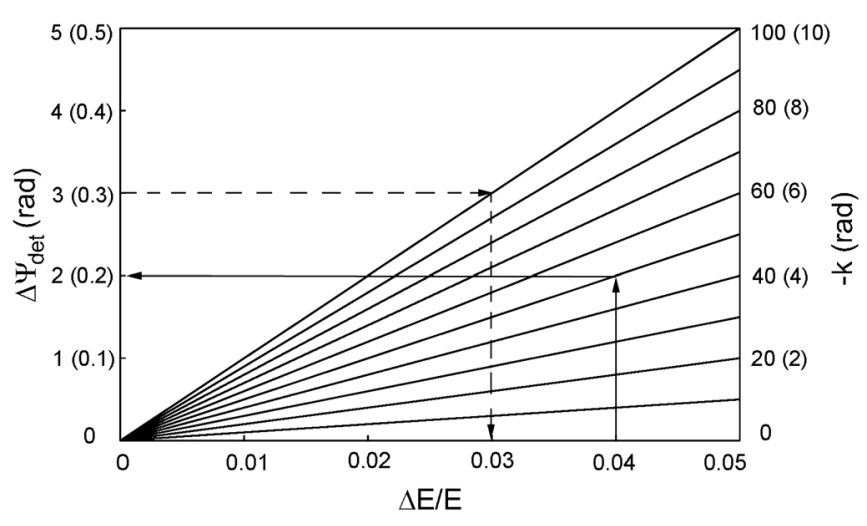

FIG. 5. The dependence of bunch phase advance $\Delta \Psi_{\text {det }}$ as a function of bunch relative energy change $\Delta E / E$ as per Eq. (7). The numbers in brackets corresponds to vertical axis scaled down by a factor of 10 . The parameter $k$ is shown as a number next to the right vertical axis. The two solid lines with arrow heads show graphically how to determine $\Delta \Psi_{\text {det }}$ if parameters $\Delta E / E$ and $k$ are known. The two dashed lines with arrow heads illustrate how to define $\Delta E / E$ if the parameters $\Delta \Psi_{\text {det }}$ and $k$ are identified.

phase advance $\Delta \Psi_{\text {det }}$ as a function of bunch relative energy gain $\Delta E / E$ is shown in Fig. 5.

The numbers shown next to the right vertical axis are the parameters $k$ from expression (7). The two arrow head straight solid lines illustrate how to calculate $\Delta \Psi_{\text {det }}$ if $\Delta E / E$ and $k$ are known. In this specific example, the vertical line is drawn up at $\Delta E / E=0.04$ until intersection with corresponding gradient line $-k=50$ (or 5). The horizontal line continued to the left defines $\Delta \Psi_{\text {det }}=2$ or $0.2 \mathrm{rad}$. The two dashed lines provide an example of calculating $\Delta E / E$ if $\Delta \Psi_{\operatorname{det}}$ and $k$ are known. In this specific instance, the horizontal line is drawn at $\Delta \Psi_{\text {det }}=3$ or $0.3 \mathrm{rad}$ to the right until intersection with corresponding gradient line $-k=100$ (or 10). The vertical line is drawn afterwards down to define value of $\Delta E / E=0.03$.

\section{RESULTS}

\section{A. Major source of microphonic noise and its minimization}

The calibrated frequency error signal $\Delta F$ is the frequency difference between the self-excited loop (SEL) and the reference frequency. This analogue signal is available as an output from low level rf (LLRF) controller as dynamic signature of resonator mechanical frequency excursions.

The major sources producing mechanical noise are as follows: (i) Slow helium gas pressure variations due to cryoplant operation; (ii) Cryogenic liquid disturbance during cryostat refill; (iii) Gas helium pressure correlation in the cryostat vessel with pressure changes in the gas return lines or other cryostats.

During the commissioning stage of linac operation, fast pressure instabilities with the frequency range $\sim 25-100 \mathrm{~Hz}$ due to pressure-acoustic oscillations (PAO) were common,

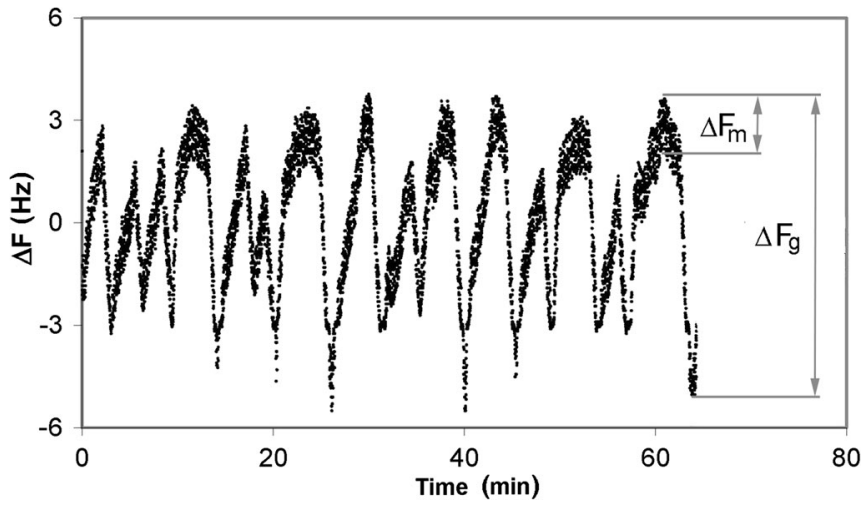

FIG. 6. Time dependence of the instantaneous resonance frequency deviation $\Delta F$ from the reference frequency of the unlocked SLR at $4.2 \mathrm{~K}$ in the ambient cryostat environment.

as described in [1]. The PAO have been observed in the LHe level sensor and in the return gas transfer tube on the cryogenic valve box. The problem was solved by installation of a damper baffle on the level sensor and a stainless steel mesh interceptor on helium gas transfer tubes.

Figure 6 shows experimental data on the time dependence of the instantaneous resonance frequency deviation from the reference frequency of the unlocked resonator in the ambient cryostat environment.

The variation of gas helium pressure causes two main effects. First is the change of the boiling temperature of liquid helium, which in turn affects the resonance frequency. The second is the displacement of the loading arm which has an important bearing on the loading capacitance of the drift tube. This results in variation of resonance frequency. The overall sensitivity of the SLR has been measured to be $1.6 \mathrm{~Hz} / \mathrm{mBar}$.

\section{B. Cavity tuning and phase lock procedures}

After conditioning at low and high rf power, all cavities were overcoupled, tuned and locked at nominal absorbed rf power of about 6 Watts per cavity. The procedure was carried out when all cavities were operated simultaneously at nominal cryogenic load for at least a few hours. In this case the entire LINAC cryogenic system was able to achieve steady state conditions in terms of gas helium pressure and temperature distributions. Therefore the phase lock conditions were optimized for the entire LINAC running at full energy gain rather than for a single cavity. Then the cavities were turned off by switching the rf amplifiers to standby mode. The DC and bunched beams were then tuned through the linac. Finally, the cavities were turned on and locked, one by one, without changing any settings in the rf control loops.

\section{Minimum detected beam current and phase resolution}

Figure 7 shows an example of the signal produced by a superconducting SLR overcoupled to the loading constant 


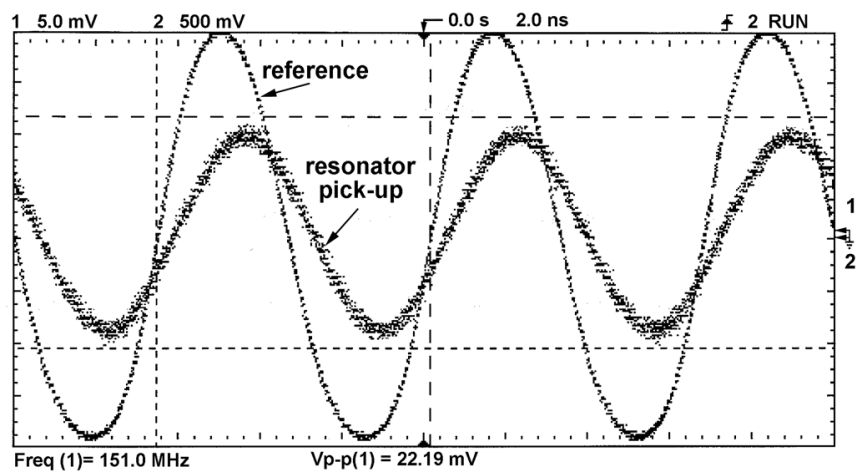

FIG. 7. Example of SLR rf field, generated by a bunched beam passing through the resonator. The energy of the ${ }^{58} \mathrm{Ni}^{+22}$ beam is $185 \mathrm{MeV}$ and the beam current is $7 \mathrm{nA}$. The last (12th) linac resonator is used as the phase detector.

$\beta \sim 101$. The signal is extracted from the coupler line and displayed on the screen of a Hewlett Packard oscilloscope $54522 \mathrm{~A}$. The linac reference signal is displayed on the same screen. The frequency of incoming ion bunches is $9.375 \mathrm{MHz}$ dictated by the operation frequency of low energy bunching system. The phase detector cavity rings at the 16th harmonic of the excitation signal.

The SLR's excitation signal is independent of the energy and atomic number of the incident ion and cross section of the passing beam bunch. The measured RMS noise level in the oscilloscope electronic circuit is typically $U_{\mathrm{Nrms}} \sim 2 \mathrm{mV}$. An acceptable signal to noise ratio SNR should be greater than $0 \mathrm{~dB}$ in order to isolate a desired signal $U_{\text {Srms }}$ from the noise floor $U_{\text {Nrms }}$. For $185 \mathrm{MeV}$ ${ }^{58} \mathrm{Ni}^{+22}$ beam the minimum beam current resolved with the oscilloscope is $I_{\mathrm{OSC} \min }=I_{b} U_{\mathrm{Nrms}} / U_{p-p}=0.6 \mathrm{nA}$, where $I_{b}=7 \mathrm{nA}$ is the average beam current and $U_{p-p}=22.2 \mathrm{mV}$ is the peak to peak value of the pickup voltage as shown in Fig. 7.

The model $8405 \mathrm{~A}$ vector voltmeter provides a measurement of rf amplitude at least from $0.1 \mathrm{mV}$ for the $B$ channel. Therefore the minimum current resolved with the vector voltmeter is at least one order of magnitude better compared to oscilloscope measurement.

Beam steering by the accelerating resonator can create an error during the measurement of the bunch phase. In the SLR it is caused by transverse magnetic and electric field components, described in the Appendix. In the small steering angle approximation, the maximum variation of transit time of a beam bunch traveling from the accelerating cavity to the phase detecting resonator is given by the equation $\Delta \tau=0.25 D_{a}^{2} /(\beta c L)$, where $D_{a}$ is the diameter of aperture in the drift tube, $c$ is the velocity of light and $L$ is the distance between the accelerating and phase detector resonators. Substituting values for $D_{a}=19 \mathrm{~mm}, \beta=0.1$ and $L=0.5 \mathrm{~m}$, the value for $\Delta \tau$ becomes $3.0 \mathrm{ps}$. The corresponding phase advance due to beam steering is

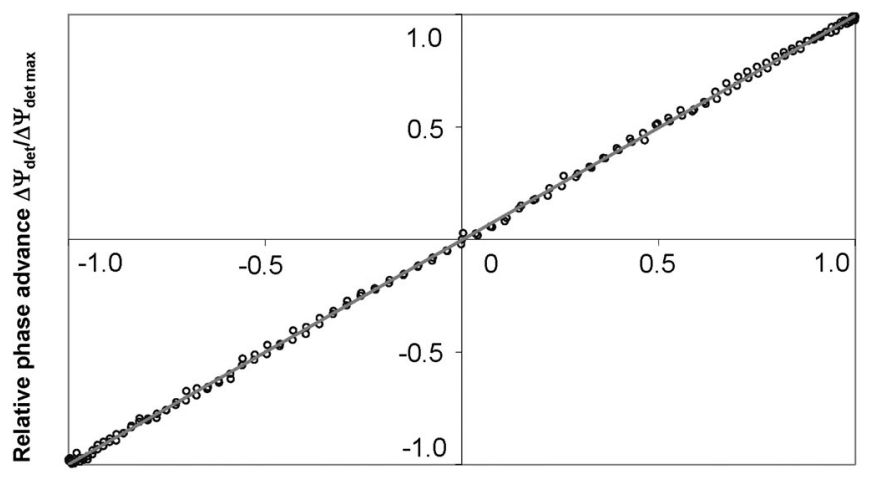

Sine of accelerating resonator phase $\sin \left(\Delta \Psi_{\text {acc }}\right)$

FIG. 8. Relative beam bunch phase $\Delta \Psi_{\text {det }} / \Delta \Psi_{\text {det max }}$ shown by open circles as detected by the superconducting phase detector as a function of the cosine of the operating phase of the linac accelerating resonator $\cos \left(\Delta \Psi_{\mathrm{acc}}\right)$. The straight solid line is the theoretical prediction of the same relationship. The energy of ${ }^{58} \mathrm{Ni}^{+22}$ beam produced by terminal and linac stripper is $185 \mathrm{MeV}$ and beam current is $7 \mathrm{nA}$. The last (12th) linac resonator is configured as the phase detector.

$\Delta \Psi_{\text {steer }}=360^{\circ} f \Delta \tau=0.16^{\circ}$, where $f=150 \mathrm{MHz}$ is the linac operation frequency.

Experimental verification of the combined effect of $185 \mathrm{MeV}{ }^{58} \mathrm{Ni}^{+22}$ beam steering and microphonic background on bunch phase measurement is shown in Fig. 8.

The experimental setup is similar to the conditions shown in Fig. 4. The beam bunch phase is detected by the 12th linac resonator, and its relative value $\Delta \Psi_{\mathrm{det}} / \Delta \Psi_{\mathrm{det} \text { max }}$ is plotted as a function of the cosine of the reference phase of the accelerating resonator $\cos \left(\Delta \Psi_{\text {acc }}\right)$. By substituting $\Delta E$ from Eq. (1) to Eq. (7), one can derive the linear dependence of $\Delta \Psi_{\text {det }} / \Delta \Psi_{\text {det max }}$ as a function of $\cos \left(\Delta \Psi_{\text {acc }}\right)$ shown as a straight line. The experimental deviation from a linear dependence (open circles in Fig. 8) is mainly caused by the combined effect of beam steering and microphonic noise. The calculated standard deviation of a signal detected by the superconducting SLR over the $360^{\circ}$ phase range of the accelerating SLR is $1.5^{\circ}$.

\section{Setting up the phases of resonators using superconducting phase detector}

Verification of the new technique was accomplished during routine linac tunes. All linac tunes were undertaken for superconducting QWR superbuncher and eleven SLRs with central frequency $\sim 150 \mathrm{MHz}$. The resonator identification was as follows: 1.1, 1.2, 1.3, 2.1-4.3, where the first number identified one of the four module cryostat. The second number identified one of the three SLRs housed in each module cryostat. All resonators were driven by SEL with amplitude and phase locked. The last linac resonator 4.3 was used in the bunch phase detection mode. The most challenging tunes were experienced with the superbuncher and the first resonators. 
This was due to the relatively long distances, $L \sim 12.3 \mathrm{~m}$ from the superbuncher to the superconducting phase detector SLR 4.3 and $L \sim 5.4 \mathrm{~m}$ from SLR 1.1 to SLR 4.3. Also the QWR and SLRs introduced beam steering which was larger at longer distances.

Figure 9 shows the plot of ${ }^{48} \mathrm{Ti}^{+19}$ beam bunch phase shift measured with superconducting SLR 4.3 with respect to the master oscillator as a function of time during entire the linac tune. The ${ }^{48} \mathrm{Ti}^{+19}$ was boosted from $170 \mathrm{MeV}$ to a final energy of $\sim 296 \mathrm{MeV}$. The beam was initially stripped to charge state $Q_{1}=11$ using the 14UD terminal stripper ( $4 \mu \mathrm{g} / \mathrm{cm}^{2}$ carbon foil), and to the final charge state of $Q_{2}=19$ with the second (high energy) stripper foil (stripper foil $8 \mu \mathrm{g} / \mathrm{cm}^{2}$ thick).

Each segment in Fig. 9 represents different operation conditions of the rf resonators along the linac: RTB in the segment highlighted in yellow is the room temperature bunching system. RTB consists of a low energy single gap buncher and two high energy choppers; SB in the segment highlighted in pink, is the superbuncher.

The area highlighted in blue represents the tuning conditions of the second SLR in the first module cryostat, resonator 1.2: (i) UNL corresponds to SLR 1.2 operating in unlocked SEL mode. Nonstabilized SEL yields significant variation of accelerating resonator phase and amplitude resulting in large deviation of phase shift measured by phase detector; (ii) PL is SLR running in SEL in phase and amplitude locked condition. Operation of accelerating resonator with stable amplitude and phase results in unchanged phase shift measured by phase detector SLR; (iii) $\phi 1$ and $\phi 2$ are two types of amplitude zero cross during crossover search, bunching and debunching, and $A \phi$ is the resonator accelerating phase.

The value of the accelerating phase $A \phi$ is set to $A \phi=\phi 1+72^{\circ}$ with respect to the bunching crossover $\phi 1$ and $A \phi=\phi 2-108^{\circ}$ with regard to debunching crossover $\phi 2$. An occasional unwelcome by-product was overbunching, this was evidenced by a wide BPM trace equivalent to a wide energy spread. To counteract this, the acceleration phase of the resonator under tune was set to $A \phi=\phi 2+108^{\circ}$ when this led to an improvement in beam profile. Typically this was necessary in 1-2 cavities across the linac.

The summary of operation conditions, corresponding to one of the typical linac tunes using the superconducting SLR phase detection technique, is listed in the Table I. The resonators identification is the same as in the explanation notes for Fig. 9. $L$ is the distance between the accelerating resonator and the phase detector SLR.

The beam energy $E$ after each successive acceleration is calculated by scaling the magnetic field $B_{180}$ of a $180^{\circ}$ achromat A2 dipole (Fig. 1) according to formula
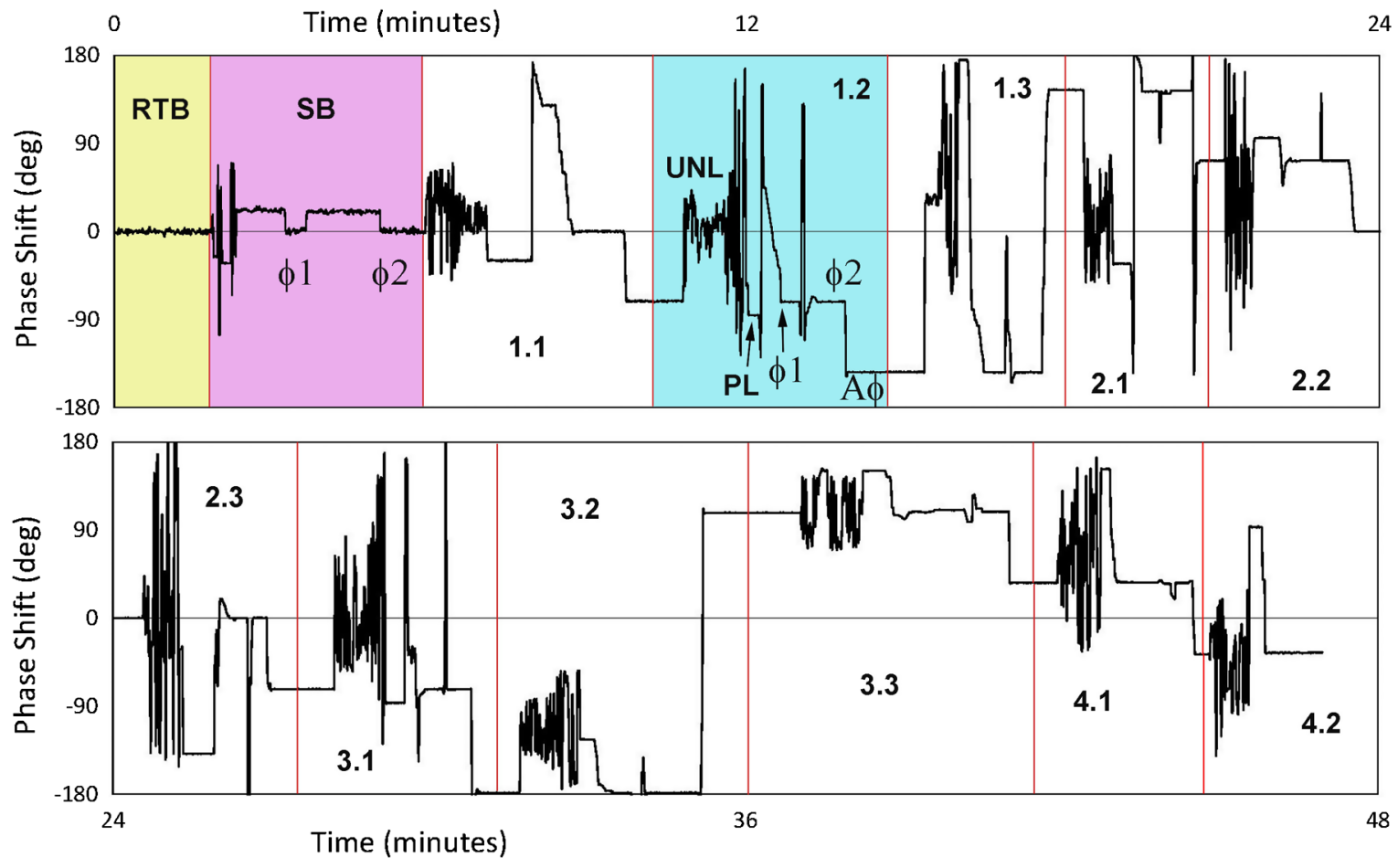

FIG. 9. Phase shift of the bunch phase measured with superconducting SLR 4.3 with respect to the master oscillator as a function of time. Each segment signifies the operation condition of the rf resonators. 1.1 stands for the first SLR in the first module cryostat, etc. RTB (yellow area) stands for room temperature buncher and SB (pink area) for super buncher. Examples of typical operating conditions of resonator 1.2: UNL- operation SEL in unlocked mode; PL- phase locked condition; $\phi 1, \phi 2$, and $A \phi$ are two crossovers and acceleration phase correspondingly. 
TABLE I. Linac tune based on superconducting cavity as phase detector.

\begin{tabular}{ccccrcc}
\hline \hline & $\begin{array}{c}E_{\mathrm{acc}} \\
R \#\end{array}$ & $\begin{array}{c}B_{180} \\
(\mathrm{MV} / \mathrm{m})\end{array}$ & $\begin{array}{c}E \\
(\mathrm{GeV})\end{array}$ & $\begin{array}{c}\Delta E \\
(\mathrm{MeV})\end{array}$ & $\begin{array}{c}L \\
(\mathrm{~m})\end{array}$ & $\begin{array}{c}E_{\text {acc } \max } \\
(\mathrm{MV} / \mathrm{m})\end{array}$ \\
\hline SB & 0.43 & 6314 & 170.0 & 0.0 & 12.34 & 0.69 \\
1.1 & 3.09 & 6533 & 182.0 & 12.0 & 5.36 & 1.49 \\
1.2 & 2.90 & 6735 & 193.4 & 11.4 & 5.07 & 1.69 \\
1.3 & 3.22 & 6955 & 206.3 & 12.9 & 4.79 & 1.95 \\
2.1 & 2.35 & 7112 & 215.7 & 9.4 & 3.77 & 2.65 \\
2.2 & 2.39 & 7268 & 225.3 & 9.6 & 3.48 & 3.06 \\
2.3 & 2.41 & 7422 & 234.9 & 9.6 & 3.19 & 3.55 \\
3.1 & 2.88 & 7601 & 246.4 & 11.5 & 2.17 & 5.63 \\
3.2 & 3.27 & 7798 & 259.3 & 12.9 & 1.88 & 7.07 \\
3.3 & 2.72 & 7957 & 270.0 & 10.7 & 1.60 & 8.89 \\
4.1 & 2.53 & 8101 & 279.8 & 9.8 & 0.58 & 26.09 \\
4.2 & 2.27 & 8227 & 288.6 & 8.8 & 0.29 & 55.09 \\
4.3 & 1.93 & 8332 & 296.0 & 7.4 & 0.0 & n/a \\
\hline \hline
\end{tabular}

$E_{\text {out }}=\left(B_{180 \text { out }} / B_{180 \text { in }}\right)^{0.5} E_{\text {in }}$ and the energy gain is $\Delta E=E_{\text {out }}-E_{\text {in }}$.

The magnetic field is measured employing Group 3 DTM-151 Digital Teslameter with an accuracy of $\pm 0.01 \%$. The average accelerating field $E_{\text {acc }}$ and maximum accelerating gradient $E_{\text {acc max }}$ for crossover phase determination are calculated using formulas (1) and (5) correspondingly.

\section{E. Determination of energy gain using superconducting phase detector}

A number of linac tunes were conducted with ${ }^{64} \mathrm{Ni}^{+20}$ and ${ }^{48} \mathrm{Ti}^{+18}$ beams with energy $E$ spanning from $160 \mathrm{MeV}$ to $352 \mathrm{MeV}$, the range of distances $L$ between accelerating cavities and phase detection resonator from $0.5 \mathrm{~m}$ to $5.4 \mathrm{~m}$ and the maximum spread of the parameter $k$ introduced in Eq. (7) from $\sim 1$ to 100 . Table II presents consolidated data of measured and calculated values of energy gain and phase shift, obtained by using the superconducting phase detector.

The beam energy gain $\Delta E_{\text {meas }}$ (after each successive acceleration) is determined by scaling the magnetic field $B_{180}$ of a $180^{\circ}$ achromat as per previous experiment.
The total phase advance $\Delta \Psi_{\text {meas }}$ observed at the phase detector cavity is measured with a setup shown in Fig. 5. $\Delta \Psi_{\text {meas }}=\Psi_{\text {acc }}-\Psi_{\text {off }}$ is proportional to the change of transit time $\Delta t_{\text {tr }}$ as per Eq. (6) corresponding to two operational conditions: (a) phase $\Psi_{\text {off }}$ is measured when the cavity under investigation is turned off and (b) phase $\Psi_{\text {acc }}$ is obtained after the same cavity is turned on at nominal accelerating field. The values of energy gain $\Delta E_{\text {calc }}$ and phase shift $\Delta \Psi_{\text {calc }}$ are calculated from Eq. (7) by substituting measured values of $\Delta \Psi_{\text {meas }}$ and $\Delta E_{\text {meas }}$ from Table II. Alternatively it can be estimated graphically from the plot presented in Fig. 8.

\section{DISCUSSION}

The phase lock procedure carried out at full nominal rf load results in highly stable operation of the linac resonators for many weeks without additional tuning required. This may imply that during linac setup at the initial stage, the first few resonators operate at a nonoptimum phase lock environment, such as gas helium pressure and equilibrium temperature distribution. However the overall settings will gradually change to optimum, when all cavities are turned on at the completion of linac setup for acceleration.

The slow variation of phase detection resonator instantaneous frequency $\Delta F_{g}$, shown in Fig. 6 , corresponds to change of the pressure in the return helium gas space with the time period of approximately $4.5 \mathrm{~min}$. The magnitude of $\Delta F_{g}$ increases when the gas helium pressure is reduced. The fast variation of $\Delta F_{m}$ is caused by microphonic noise in the frequency range of 47-56 Hz.

The SLR in both phase detection or acceleration modes is operated in overcoupled mode to the coupling constant $\beta=101$. Therefore minimum tuning has been required to switch it quickly between two operation modes. The tuning beams of sub-nA intensities has been demonstrated, as shown in Fig. 7. The typical long term peak-to-peak excursion of instantaneous resonance frequency has been measured $\Delta F_{g} \sim 8.4 \mathrm{~Hz}$. It is caused by slow gas helium pressure variations within $10 \mathrm{mBar}$ and time constant of $T_{\mathrm{GHe}} \sim 4.5 \mathrm{~min}$, Fig. 6 . Slow pressure variation would

TABLE II. Comparison of analytical and experimental determination of energy gain and phase shift acquired by using superconducting phase detector.

\begin{tabular}{llcccccc}
\hline \hline Ion beam & $E(\mathrm{MeV})$ & $L(\mathrm{~m})$ & $k(\mathrm{rad})$ & $\Delta E_{\text {meas }}(\mathrm{MeV})$ & $\Delta E_{\text {calc }}(\mathrm{MeV})$ & $\Delta \Psi_{\text {meas }}(\mathrm{rad})$ & $\Delta \Psi_{\text {calc }}(\mathrm{rad})$ \\
\hline${ }^{64} \mathrm{Ni}^{+20}$ & 351.694 & 0.58 & 8.45 & 8.80 & 8.70 & 0.209 & 0.211 \\
${ }^{48} \mathrm{Ti}^{+18}$ & 216.05 & 1.88 & 30.26 & 11.72 & 11.84 & 1.658 & 1.642 \\
${ }^{64} \mathrm{Ni}^{+20}$ & 318.5 & 3.19 & 48.83 & 9.26 & 9.33 & 1.431 & 1.42 \\
${ }^{48} \mathrm{Ti}^{+18}$ & 207.13 & 3.19 & 52.44 & 8.92 & 8.82 & 2.234 & 2.259 \\
${ }^{64} \mathrm{Ni}^{+20}$ & 308.8 & 3.48 & 54.1 & 9.70 & 9.76 & 1.71 & 1.699 \\
${ }^{48} \mathrm{Ti}^{+18}$ & 199.09 & 3.48 & 58.35 & 8.04 & 8.1 & 2.374 & 2.357 \\
${ }^{64} \mathrm{Ni}^{+20}$ & 299.963 & 3.77 & 59.46 & 8.84 & 8.72 & 1.728 & 1.744 \\
${ }^{64} \mathrm{Ni}^{+20}$ & 278.24 & 5.07 & 83.04 & 10.76 & 10.86 & 3.241 & 3.211 \\
${ }^{48} \mathrm{Ti}^{+18}$ & 159.75 & 5.36 & 100.33 & 10.38 & 10.51 & 6.602 & 6.517 \\
\hline \hline
\end{tabular}


affect the output signal from pick up port. Assuming $\Delta F_{g} \ll f_{0}$ the phase of pick up signal will change as per Eq. (8) below:

$$
\Delta \Psi=\tan ^{-1}\left(2 Q_{L} \Delta F_{g} / f_{0}\right)
$$

For example, the phase would be drifting in the range $\Delta \Psi_{g}=6.4$ degrees. The peak-to-peak value of fast $\sim 50 \mathrm{~Hz}$ SLR frequency excursion, caused by microphonic noise, is typically $\Delta F_{m} \sim 1.8 \mathrm{~Hz}$, which according to Eq. (8) corresponds to phase jitter of $\Delta \Psi_{m}=1.4$ degrees at $f_{0}=150 \mathrm{MHz}$. Microphonic frequency excursions remain the main contributor to phase error output compared to helium gas pressure variation, since the measurement is accomplished in a time frame much shorter than $T_{\mathrm{GHe}}$.

The second source of phase error is caused by beam steering during bunch acceleration. The measured standard deviation of detected phase over $360^{\circ}$ phase range, Fig. 8, is $\Delta \Psi_{m+s}=1.5^{\circ}$. This value consolidates the combined effects of microphonic noise and beam steering. The RMS phase error caused by microphonics or steering corresponds to the measured standard deviation since the input signal has zero mean. Therefore the contribution of beam steering can be estimated from the equation for RMS combinations $\Delta \Psi_{s}=\left(\Delta \Psi_{m+s}^{2}-\Delta \Psi_{m}^{2}\right)^{1 / 2}=0.5$ degrees. During crossover search the phase error contribution from the beam steering effect can be neglected as it is not significant at low accelerating field. However it has to be accounted for throughout determination of energy gain.

The overall phase resolution can be further improved by the following techniques: (i) More overcoupling, however this results in lower sensitivity to beam intensity; (ii) Averaging of the phase jitter signal as it consists of a dominant single frequency corresponding to one of the mechanical resonance mode of the cavity; (iii) Synchronization to slow variation of gas helium pressure in order to take phase measurements at the same pressure; (iv) Using as the phase detector cavity a resonator with the best mechanical stability. For example the resonance frequency sensitivity to helium gas pressure of Nb-sputtered OHFC QWR is nearly 4 times less than a SLR. (v) Any cavity along the linac structure can be used as a phase detector by multiplexing its output to the same electronic device as described in [5]. In spite of increased cost and complexity, this option reduces the error associated with long drift distances between the accelerating resonator and the phase detector resonator.

The phase detection technique based on a superconducting resonator allows better resolution for resonator crossover phase $\phi 1$ and $\phi 2$, acceleration phase $A \phi$ and better sensitivity to accelerating current when compared to the BPM technique described in [9]. The high precision accelerating phase setting does not result in higher beam energy gain or improved transmission. Therefore, the main application of this technique is when fast tuning beams of ultra-low, sub-nA, intensities is required.
According to Table I, the maximum accelerating field should be kept below $E_{\text {acc max }}$ in the first three resonators in order to obtain unambiguous beam crossover phase data. This condition is specific for the linac configuration, discussed in this paper, can be easily evaluated for any linac configuration using Eq. (5). The second before last resonator 4.2 should be also set at much lower power $\sim 10 \%$ of nominal accelerating field. At nominal field the crosstalk between resonators 4.2 and 4.3 arises through beam ports and causes phase detector ringing from the wrong excitation source, rather than the beam bunches.

A wide range of tunes, with a range of the parameter $k$ from $\sim 1$ to 100 has been accomplished for energy gain and phase advance determination with accuracy better than $1 \%$ as shown in Table II. Energy gain resolution may be further improved by beam resteering after each successive acceleration. For the best results it is accomplished by inserting an aperture with a diameter of $2 \mathrm{~mm}$ at the linac exit.

Superconducting phase detector operation turns any accelerating cavity into a beam phase detector or energy detector. It is less sensitive to beam steering and defocusing with energy changes as compared to the BPM technique [9].

This new technique provides good accuracy for determination of beam bunch phase and provides a record of a specific linac tune. More importantly it does not require an energy dispersion bend, and so can be used in straight sections of a linac facility. It improves overall operation capability resulting in considerable enhancement in linac performance and its availability for operation, in particular with low beam intensity. The same technique can be used to produce a table of parameters for setting resonator phases to precalculated values.

\section{ACKNOWLEDGMENTS}

This work has been supported by the Australian Federal Government Superscience/EIF funding under the NCRIS mechanism. The author wishes to express his appreciation to RSPE's Nuclear Physics technical staff members P. Linardakis, T. Tunningley, D. Tsifakis, A. Muirhead, J. Heighway and J. Bockwinkel for their skill and diligence during the cryogenic plant and linac operations. Benoit Gay is specially thanked for his help with STRAND7 mechanical modes simulations. The author thanks his colleague from the Department of Nuclear Physics, Dr Tibor Kibedi, who provided insights and expertise in cryogenics, greatly assisting in linac operations. The author is grateful to Prof. D. Hinde for his stimulating remarks.

\section{APPENDIX: BEAM STEERING CAUSED BY ACCELERATING SLR}

The distributions of electrical field $E_{y}$ and $E_{x}$ normalized to the peak electrical field $E_{p}$ on the axis of the SLR are shown in Fig. 10. $X$ and $Y$ coordinates are in horizontal and 


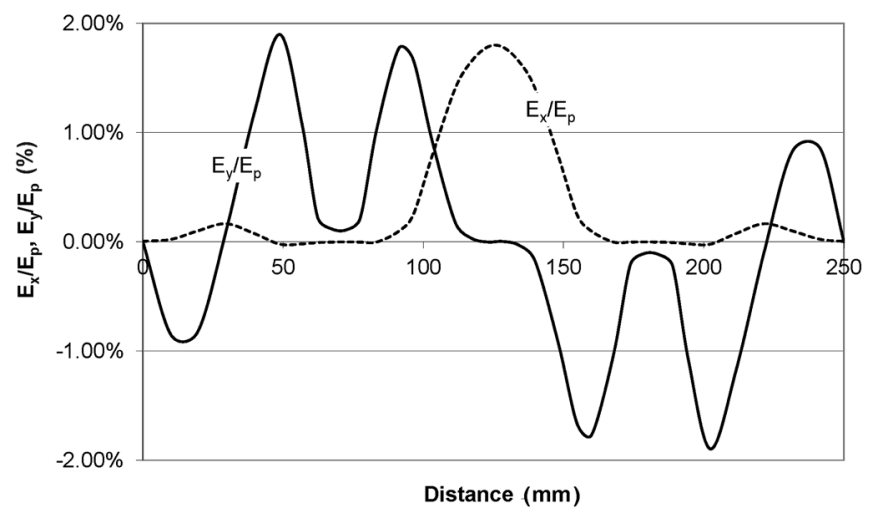

FIG. 10. MWS $E$-field distribution along the axis of the $150 \mathrm{MHz}$ SLR.

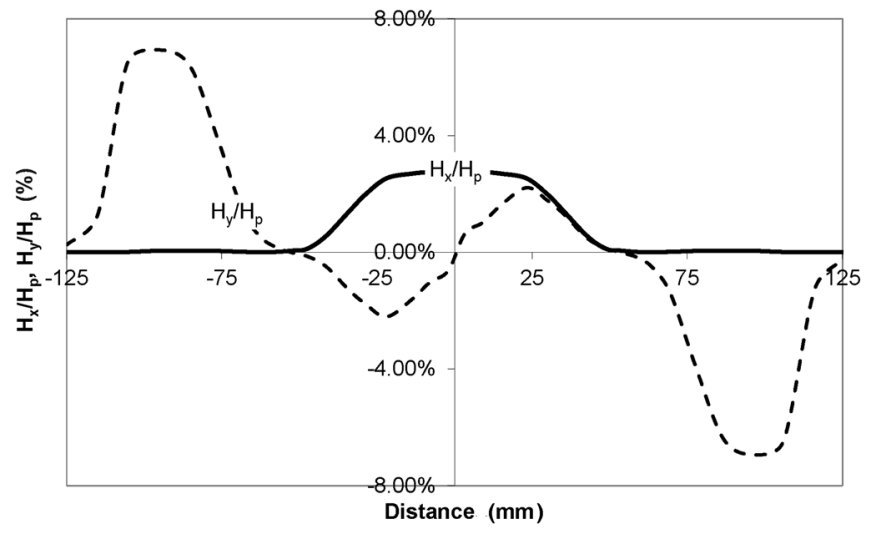

FIG. 11. MWS $H$-field distribution along the axis of the $150 \mathrm{MHz}$ SLR.

vertical directions respectively. The $Z$ coordinate coincides with the ion beam axis.

The horizontal steering effect due to $E_{x}$ mostly in the inner gap may produce horizontal steering due to nonsymmetry of the SLR loops in the $X$-plane. The described $E$-field steering in the SLR is quite different to $E$-field steering in a QWR where the $E_{x}$ component is not present. Nevertheless the steering strength, proportional to $E_{x, y} / E_{p} \approx 1-2 \%$, is comparable in both SLR and QWR.

The distributions of $H_{x}$ and $H_{y}$ normalized to peak magnetic field $H_{p}$ on the axis of the SLR are shown in Fig. 11. Once again it is very different in a SLR compare to a QWR, where the only $H_{x}$ is present steering in the vertical plane.

The surface current oscillating between loop arms and in the loop's valley, mainly in the $y$-direction, creates the magnetic field in the middle gap. This enhances the $H_{x}$ component of the $H$-field. On the contrary, the magnetic field in the outer gaps is created by surface current oscillating between loop arms and the can wall. The opposite going currents in the can walls connect more in the wall in $X-, Z$ - direction and need not return to the stub assembly through the joint. This enhances the $H_{y}$ component of the $H$-field in outer gap. The $H_{x}$ in the inner gap produces vertical steering. $H_{y}$ causes steering in the horizontal plane in the outer gaps. The electromagnetic steering is a function of rf phase and does not exist at crossover phase. The simplest technique to compensate for beam steering is to offset the cavity in the vertical and horizontal direction with respect to the beam axis. The optimum cavity offset can be found by beam transport simulation.

[1] D. C. Weisser and N. R. Lobanov, Linac boosters-an overview, in Proceedings of 8th International Conference on Heavy ion Accelerator Technology, edited by K. Shepard, AIP Conf. Proc. No. 473 (AIP, Woodbury, NY, 1999), pp. 117-137.

[2] M. Sugitani, M. Tsukihara, S. Takei, E. McIntyre, D. DeLuca, and T. Parrill, Introducing the NV-GSD-HE3, a new high energy implanter, in Proceedings of the 12th International Conference on Ion Implantation Technology, Kyoto (IEEE, Piscataway, NJ, 1998), p. 192.

[3] S. Canella, An automatic procedure to find and set the shift phase for the superconducting resonators in the ALPI accelerator, in Proceedings of the 1997 International Conference on Accelerator and Large Experimental Physics Control Systems, Beijing, China (Science Press, Beijing, 1997), p. 491.

[4] M. Bellato, A. Dainelli, and M. Poggi, Design and tests for the fast Faraday cup of the ALPI post-accelerator, Nucl. Instrum. Methods Phys. Res., Sect. A 382, 118 (1996).

[5] S. I. Sharamentov, R. C. Pardo, P. N. Ostroumov, B. E. Clifft, and G. P. Zinkann, Superconducting resonator used as a beam phase detector, Phys. Rev. ST Accel. Beams 6, 052802 (2003).

[6] N. R. Lobanov, P. Linardakis, and D. Tsifakis, Tuning the linac with superconducting resonator used as a phase detector, in Proceedings of the 17th Workshop on RF Superconductivity, Whistler, Canada 2015 (JACoW, Geneva, 2015), p. TUPB024.

[7] S. Takeuchi and K. W. Shepard, A sensitive beam-bunch phase detector, Nucl. Instrum. Methods Phys. Res., Sect. A 227, 217 (1984).

[8] A. Facco, K. Rudolf, G. Bassato, and A. Battistella, A high sensitivity phase stabilizing system and beam current monitor, Nucl. Instrum. Methods Phys. Res., Sect. A 337, 253 (1994).

[9] N. R. Lobanov, P. Linardakis, and D. Tsifakis, Tuning the superconducting linac at low beam intensities, in Proceedings of the 17th Workshop on RF Superconductivity, Whistler, Canada 2015 (JACoW, Geneva, 2015), p. TUPB025. 